\title{
III.
}

\section{Zur Wirkung des ultravioletten Lichtes auf die Zelle.}

(Aus der Deutschen Dermatologischen Klinik in Prag.)

Von

Professor C. Kreibich.

In Untersuchungen über die Wirkung des ultravioletten Lichtes auf intrazelluläre Fermente im Eiterausstrich (Arch. f. Derm. u. Syph. 113) ließ sich neben der Zerstörung der Oxydase und Peroxydase eine Verzögerung in der Zerlegung von $\mathrm{H}_{2} \mathrm{O}_{2}$, also eine Verlangsamung der Katalasenwirkung nachweisen, gleichzeitig damit trat eine stärkere Protoplasmafärbbarkeit in der belichteten Seite des Ausstriches ein. Dieselbe war nicht bedingt durch Wärmewirkung oder durch eine Veränderung in der Alkaleszenz, es bestand auch keine Erschöpfung oder Zerstörung der Katalase bei der betreffenden Versuchsanordnung. Als Grund für die bessere Färbbarkeit des Protoplasmas mußte eine physikalisch-chemische Veränderung des Protoplasmas angenommen werden, derzufolge die an das Protoplasma gebundene Katalase nach der Belichtung nur in verlangsamter Weise zur Wirkung gelangt. Darnach war es weiter von Interesse, die Wirkung des ultravioletten Lichtes auf den Zellverband zu studieren. v. HeB sah nach Einwirkung des ultravioletten Lichtes zunächst Zellteilung, später Kerndegeneration in dem Kapselepithel der Linse auftreten, wir wählten für die folgenden Versuche aus der Ernährung ausgeschaltetes supravitales Gewebe, und zwar die Kornea von frisch geschlachteten Rindern. Damit wurden zwei im Wesen verschiedene Versuche angestellt: I. Es wurden Streifen von Rinderkornea über Agar 12-24. Stunden im Thermostaten gehalten, hierauf wurde eine Hälfte mit Staniol bedeckt und die andere Hälfte 15-20' mittels künstlicher Höhensonne bei $30 \mathrm{~cm}$ Abstand bestrahlt. Nach der Bestrahlung sind beide Hälften makroskopisch gleich aussehend. Kommen die Stücke in $80 \%$ Alkohol oder in Zenkersche Flüssigkeit, so wird die unbestrahlte Seite rasch opak weiß, während die bestrahlte Hälfte viel länger durchscheinend bleibt und sich erst später trübt. Im Schnitt zeigt die unbestrahlte Hälfte jene Veränderungen, wie ich sie anderwärts beschrieben habe. Die gleiche Beschaffenheit zeigt auch die basale Hälfte der bestrahlten Seite. Von ihr horizontal, ferner scharf an der Staniolgrenze abgesetzt, liegt die durch die Belichtung erzeugte Veränderung. In dieser Zone sind die Kerne kleiner, etwas geschrumpft, ihr Chromatin zusammengedrängt, daher der Kern intensiver gefärbt. Die wesentliche Veränderung aber liegt im Protoplasma, welches sich sehr intensiv mit Protoplasmafarben färbt. Nach van Gieson ist die Partie intensiv rot, desgleichen mit Eosin, auch mit Methylgrünpyronin hebt sich durch deutlichere Rotfärbung des Protoplasmas der Streifen ab, während er mit Methylenblau gleichmäßig blau gefärbt ist. Mit Immersion sieht man das ganze Protoplasma der Zelle 
gefärbt und die Zelle erscheint deutlich in ihrer polygonalen Begrenzung, im Vergleich zur unbelichteten Seite gleichsam wie fixiert. Die Grenze ist selbst mit der Immersion noch eine scharfe. Am ungefärbten Schnitt ist die veränderte Stelle für Licht durchgängig (in Glyzerin oder Wasser gesehen), dementsprechend erseheint der veränderte Streifen im Dunkelveld dunkel. Veränderungen, die übereinstimmen mit dem später eintretenden Opakwerden in Alkohol und Zenkex. Die der Belichtung vorausgehende Agarkultur hatte in dem Falle nur den Zweck, das Gewebe zur Quellung zu bringen und die Lichtveränderung an dem breiter gewordenen Korneaepithel anschaulicher zu gestalten. Die geschilderten Veränderungen haben eine gewisse Ahnlichkeit mit der Einwirkung von Tannin auf Gewebe.

Der II. Versuch bestand in folgendem: Es wurden Streifen der Kornea zur Hälfte in obiger Weise durch $15-20^{\prime}$ bei $30 \mathrm{~cm}$ Abstand mit der künstlichen Höhensonne bestrahlt und kamen dann 8, 24, 36 Stunden auf Agar in den Thermostaten. Bereits nach 8 , deutlicher nach 24 Stunden, ist die bestrahlte Hälfte opak trïb, während die unbestrahlte durchscheinend bleibt. Fixiert man rasch nach Gehuchten, so erscheint die obere Hälfte der unbestrahlten Seite durch die rasche Fixation ziemlich intensiv sowohl im Kern, wie auch im Protoplasma gefärbt, während die Kerne der unteren Hälfte in ihrem Chromatinnetz gut erhalten sind und auch das Protoplasma die ursprüngliche Zellform erhalten hat. Auf der bestrahlten Seite ist nur an der Oberfläche ein ganz dünner Streifen ähnlich wie auf der geschützten Hälfte fixiert und intensiver gefärbt, die darunter gelegene übrige Partie der Kornea weicht im Aussehen stark von der unbestrahlten Seite ab. Das wesentlichste ist die homogene Schrumpfung der Kerne zu runden, intensiv gefärbten strukturlosen Kugeln, der Zerfall des Chromatin zu intensiv gefärbten Schollen. Ist das Agar feucht (Kondenswasser), so sind die Zellen stark auseinandergezogen. Bei trockenerem Agar sicht man zwischen obigen Zellen solche, deren Kerne sich mit polychromem Methylenblau blaß bläulich färben, keine Struktur mehr aufweisen; in diesen Zellen ist auch in verschiedenem AusmaB das Protoplasma homogen und blaß bläulich gefärbt. Solche Zellen erscheinen ähnlich wie der oberste Streifen im Dunkelfeld dunkel, sind für Licht besser durchgängig und somit als hyalin degenerierte zu erkennen.

Die am Korneaepithel erzielten lichtveränderungen ergänzen die eingangs geschilderten Veränderungen am Leukozytenprotoplasma. Das Verständnis für den physikalisch-chemischen Vorgang nach Einwirkung von ultraviolettem Licht ergibt sich aus den Arbeiten von Dreyer, Hansen, Chalupecky und Schanz. Dreyer und Hansen fanden, daß nicht lösliche Eiweißkörper in unlösliche übergeführt werden. Chalupecky konnte am Eiereiweiß und Linseneiweiß zeigen, daß Albumin, bevor es koaguliert, in Globulin übergeführt wird. Schanz erbrachte am Serumeiweiß den gleichen Nachweis und leitet aus den bisherigen Versuchen den Satz ab: „Das Licht verändert die Struktur der EiweiBkörper in dem Sinne, daß aus leicht löslichen schwerlösliche werden." 
Mit dieser Umwandlung der Eiweißkörper geht nun in der Zelle einher die stärkere Bindung der Katalase und verzögerte Katalasenwirkung, die deutlichere Protoplasmafärbung, ferner Veränderungen am Kern und Protoplasma, die nach Einwirkung dem lebenden Körper nachgeahmter Verhältnisse (Wärme und Feuchtigkeit) zur homogenen Schrumpfung des Kernes Pyknose, und schließlich zur hyalinen Degeneration der Zelle führen.

\title{
IV. \\ Zur Kenntnis des Leberinfarktes.
}

\author{
Von \\ Prof. Dr. Richard Kretz, \\ Privatdozent für pathologische Anatomie an der Universität Wien, \\ zurzeit Pathologe am Kriegsspital Nr. 2 in Wien.
}

(Hierzu 2 Textfiguren.)

Die anämische Nekrose der Leber folgt bekanntlich nur der Ausschaltung des Blutzuflusses aus der Leberarterie, nicht dem der viel mächtigeren Pfortaderzirkulation. Der Lebergewebstod infolge Fehlens der arteriellen Blutzufuhr aus der im Verhältnis zur Organgröße sehr bescheidenen Arterie, die aber als ernährendes Gefäß der Leberzellen große Wichtigkeit besitzt, ist kein besonders häufiger Befund am Obduktionstische; unter etwa 18000 Sektionsfällen erinnere ich mich an etwa ein Dutzend makroskopisch erkannter anämischer Lebernekrosen. Weitaus die mächtigste anämische Lebernekrose sah ich nach operativer Unterbindung des rechten Leberarterienastes: es waren ungefähr zwei Drittel des Organes nekrotisch geworden. Am öftesten sah ich die anämische Nekrose an den Rändern von nicht ganz frischen Leberzerreißungen; der Befund ist wohl ohne besondere klinische Bedeutung, weil fast immer das primäre Trauma der Zerreißung den Verlauf des Falles bedingt. Die dritte Ursache von kleinen bis mittelgroßen Infarkten stellt die eigentliche Embolie der Leberarterie dar; sie ist ziemlich selten, ein besonders schöner Fall dieser Art soll im folgenden beschrieben werden:

Der Fall stammt von einem 30 jährigen Infanteristen (im Zivilberufe Monteur), der am 18. November 1915 mit einer schweren akuten Endokarditis zur Obduktion gelangte. Die Endokarditis hatte sich in einem von früheren Veränderungen nicht ganz freien Herzen im Anschluß an eine Halsentzündung mit stärkerer Halslymphdrüsenentzündung entwickelt; der freie Rand der Mitralklappe war im ganzen. Umfang mit sehr zahlreichen feinzottigen Auflagerungen bedeckt, die sich 